\title{
Extending Catmull-Clark Subdivision and PCCM with Polar Structures
}

\author{
Ashish Myles \\ University of Florida \\ amyles@cise.ufl.edu
}

\author{
Kestutis Karčiauskas \\ Vilnius University \\ kestutis.karciauskas@mif.vu.lt
}

\author{
Jörg Peters \\ University of Florida \\ jorg@cise.ufl.edu
}

\begin{abstract}
We complete and bring together two pairs of surface constructions that use polynomial pieces of degree $(3,3)$ to associate a smooth surface with a mesh. The two pairs complement each other in that one extends the subdivisionmodeling paradigm, the other the NURBS patch approach to free-form modeling.

Both Catmull-Clark [3] and polar subdivision [7] generalize bi-cubic spline subdivision. Together, they form a powerful combination for smooth object design: while CatmullClark subdivision is more suitable where few facets join, polar subdivision nicely models regions where many facets join, as when capping extruded features. We show how to easily combine the meshes of these two generalizations of bi-cubic spline subdivision.

A related but different generalization of bi-cubic splines is to model non-tensor-product configurations by a finite set of smoothly connected bi-cubic patches. PCCM [12] does so for layouts where Catmull-Clark would apply. We show that a single NURBS patch can be used where polar subdivision would be applied. This spline is singularly parametrized, but, using a novel technique, we show that the surface is $C^{1}$ and has bounded curvatures.
\end{abstract}

\section{Motivation, Literature and Overview}

While the mesh-based subdivision representation yields an intuitive visualization for interactive modeling, surfaces constructed from finitely many NURBS patches are preferred in CAD packages and convenient for GPU implementations. Here, we show how four bi-cubic options (see Table 1) coexist and complement each other. Generalized

\section{Table 1. Bi-cubic surface constructions}

\begin{tabular}{l|ll}
\hline patches & quadrilateral & polar \\
\hline subdivision & Catmull-Clark [3] & bi-cubic polar [7] \\
finite & PCCM [12] & new (Section 4)
\end{tabular}

bi-cubic subdivision is typically associated with Catmull-
Clark subdivision [3]. Catmull-Clark subdivision is popular due to its close relation to the industry standard bi-cubic
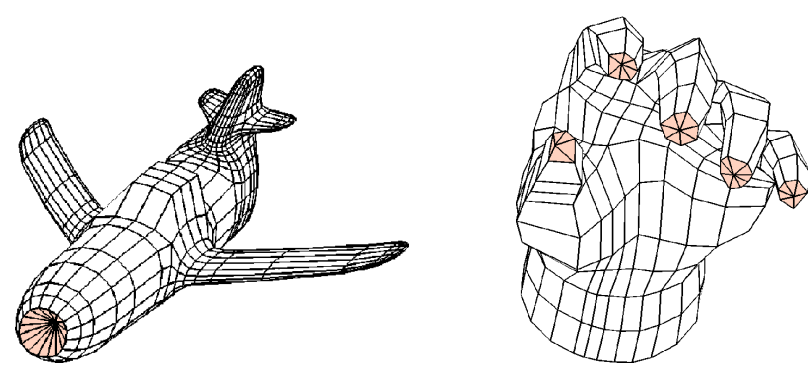

Figure 1. Augmenting Catmull-Clark meshes with polar vertices: nose of airplane (left) and tips of fingers (right).

tensor-product splines or NURBS. However, where many facets join (high valence $n$ ) or where features are extruded, the resulting shape can be poor and show unintended ripples (see e.g. Figure 2). Moreover, Catmull-Clark is prone to generating saddle points in the limit even though the control net is designed for convex shape [8]. The second problem has recently been addressed in $[9,4,1]$ and suppressing macroscopic ripples arising in the first subdivision step motivated the global shape optimization in [5]. In [6], it was argued that both ripple and saddle problems can be resolved by switching to a polar layout of facets. A neighborhood of a high-valence vertex (Figure 3, top of mushroom, finger tips) has polar layout if the vertex is surrounded by one layer of triangles while the remaining mesh consists of quadrilateral facets, with always four joining at a vertex (formal definitions follow in Section 1.1). Polar layout naturally appears in the design of surfaces of revolution. Recently, in [7], a simple generalization of bi-cubic subdivision to polar layouts was proposed. Polar meshes can also be refined using the more general tool of quad-tri subdivision $[19,18]$; but polar subdivision [7] is preferable for polar layout as it generates a finite number of bi-cubic patches in the surface corresponding to the transition from a quad to a trian- 


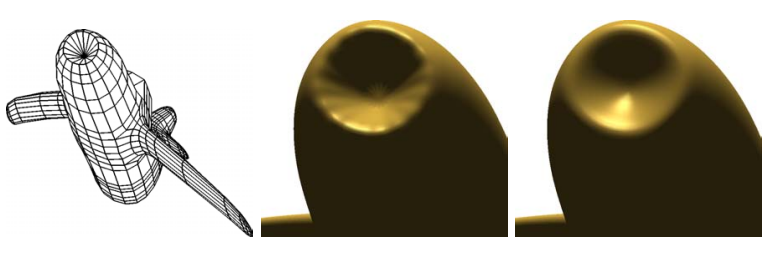

Figure 2. (left) Airplane with polar nose. (middle) Catmull-Clark subdivision generates ripples, whereas (right) polar subdivision looks natural.

gle facet while $[19,18]$ creates infinitely many, half of them three-sided. Also for the polar layout, Jet Subdivision [6] generates $C^{2}$ surfaces with good curvature distribution. But the construction is more complex and of degree $(6,5)$ and therefore outside of our focus on bi-cubic schemes. Similarly, replacing Catmull-Clark meshes by those generated in [9] is compatible with polar subdivision but outside the focus.

Our approach for combining polar and Catmull-Clark subdivision meshes is to use Catmull-Clark subdivision except for special rules where the mesh has a polar layout. This has the advantage that any input mesh admissible for Catmull-Clark can be handled by the combined subdivision. In effect, the polar submeshes will be temporarily split off from the remaining mesh so that the same code base as for Catmull-Clark subdivision can be used and just one special subroutine for polar submeshes is added to improve the surface quality. The resulting surface pieces match exactly at their interfaces where they represent the same bi-cubic polynomial spline patches.

Our approach for complementing a finite bi-cubic spline construction with a finite polar construction is analogous and compatible with PCCM [12]. We interpret, after a few steps, the polar subdivision control mesh as the control net of a NURBS surface. This single polynomial NURBS patch has a degenerate edge and only the NURBS coefficients next to it have to be adjusted. The result is a $C^{1}$ surface with bounded curvature. Due to the growth of the regular mesh and the boundedness of the construction near irregular mesh points, refining the input mesh trivially but importantly allows switching from the subdivision mesh to the NURBS representation and vice versa with an arbitrarily small error.

In Section 2, we review and extend the recently developed bi-cubic polar subdivision and in Section 3 we show how to apply it in the context of meshes of arbitrary connectivity. In Section 4, we construct single NURBS patches for polar vertices. This is analyzed in the Appendix with the help of a novel use of new proof techniques.

\subsection{Definitions}

A control net or mesh is an embedding of a graph, in $\mathbb{R}^{3}$ with vertices identified as points and connectivity indicated by line segments. The graph is assumed to have the connectivity of a 2-manifold. The number of neighbors of a vertex is called the valence of the vertex. It is denoted by $n$. The $i$-link of a vertex consists of points that can be reached by traversing a shortest path of $i$ edges. The 0 -link consists only of the vertex itself. The $i$-layer of a vertex $\mathbf{v}$ is the collection of faces, whose closest vertex to $\mathbf{v}$ is in $\mathbf{v}$ 's $i$-link. The 0-layer of $\mathbf{v}$ consists of all its incident faces.

A polar vertex is one whose 0-layer consists only of triangles (see Figure 3). A polar structure consists of a polar vertex of valence $n \geq 6$ whose 1-layer and 2-layer consist only of quadrilateral facets (quads) and such that the vertices of the 1- and 2-link are 4-valent (see Figure 3). We will not need to consider polar rules for boundaries. A polar structure makes the term polar layout precise.

\section{Compatible polar mesh refinement}
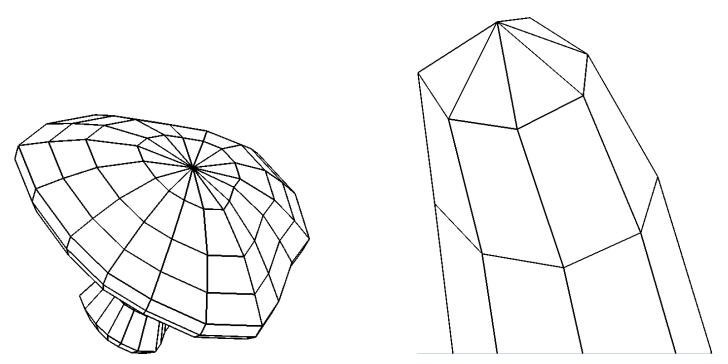

Figure 3. Polar vertices and structures.

Compatible polar mesh refinement is derived from [7] to yield a consistently $k$-times subdivided mesh when the Catmull-Clark mesh is augmented with polar structures.

Consider a mesh with the latitude-longitude connectivity of the sphere as in Figures 3, left, and 7. This yields two polar vertices. Except for constructing polar vertices and their 1-links, the refinement algorithm in [7] applies cubic spline refinement only in the longitudinal (radial) but not in the latitudinal (circular) direction. For the polar vertex and its 1-link, the stencil weights for Figure 4, left, are given as

$$
\begin{gathered}
\alpha:=\beta-\frac{1}{4}, \quad \beta:=\frac{5}{8}, \quad c_{n}^{k}:=\cos \left(\frac{2 \pi k}{n}\right), \\
\gamma_{k}:=\frac{1}{n}\left(\beta-\frac{1}{2}+\frac{5}{8} c_{n}^{k}+\left(c_{n}^{k}\right)^{2}+\frac{1}{2}\left(c_{n}^{k}\right)^{3}\right)
\end{gathered}
$$

where $n$ is the valence of the polar vertex. The analysis in [7] shows that, for $n \geq 6$, this radial subdivision results in 


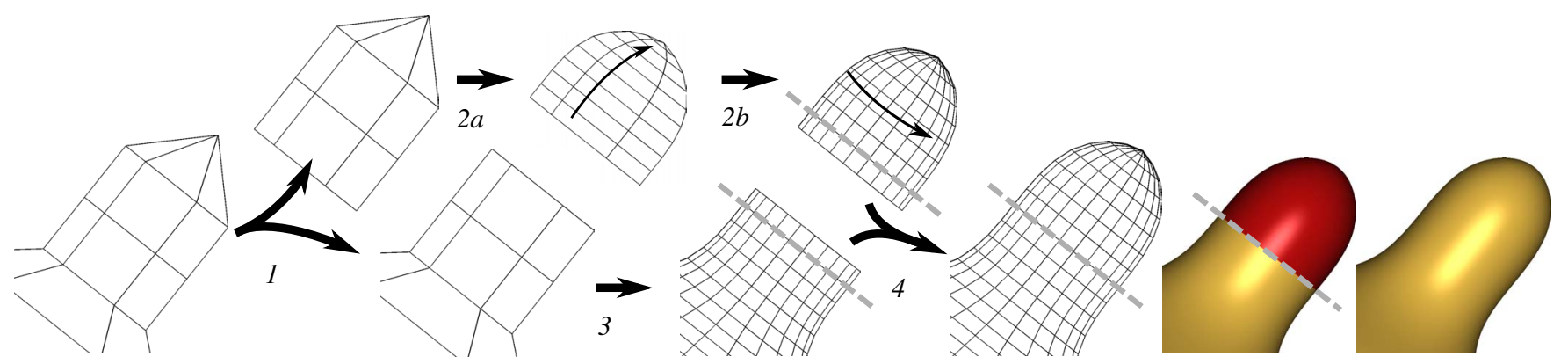

Figure 5. Generalized bi-cubic subdivision steps. (1) Separating the input mesh. (2) Subdividing the polar structure radially (2a) then circularly (2b). (3) Subdividing the remainder. (4) Joining the refined meshes after removal of overlapping facets. (right) The limit surface.

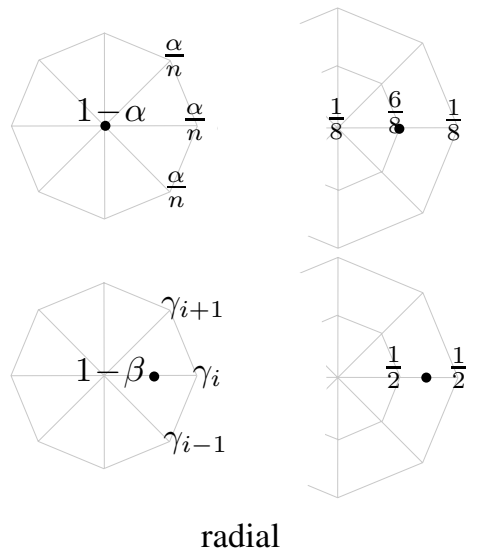

Figure 4. Refinement stencils for polar subdivision ([7]). (left) Radial subdivision at the polar vertex, (middle) radial subdivision everywhere else, (right) circular subdivision everywhere else.

an everywhere $C^{2}$ surface except at the central limit point. At the central point the surface is $C^{1}$ with bounded curvature. Moreover, the ripple and saddle artifacts of CatmullClark subdivision do not appear.

As illustrated in Figure 6, purely radial refinement results in a mismatch or a mesh with T-corners at the transition to Catmull-Clark subdivision since Catmull-Clark subdivision simultaneously subdivides radially and circularly.

In order to leverage and preserve the good results of radial subdivision and still display a consistent control net after $k$ steps, we proceed as illustrated in Figure 7: we do not alternate radial and circular subdivisions in the $k$ steps but use compatible polar mesh refinement.

(a) Apply $k$ steps of radial subdivision and save the level $k$ polar structure in case we continue subdivision later.

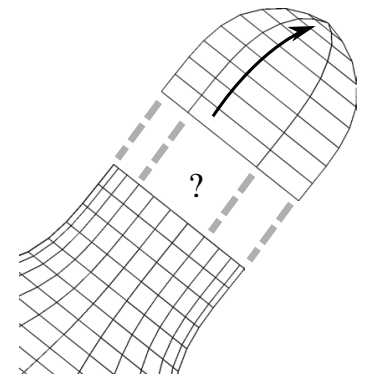

Figure 6. Mismatch on the mesh between radial subdivision ((a)) and Catmull-Clark subdivision ((b)).

(b) Apply $k$ circular subdivision steps.

Since step (a) preserves the valence and hence the analysis of reference [7], we base any continued refinement on the saved polar structure. Alternating radial and circular subdivision creates local curvature fluctuations By contrast, applying step (b) only a posteriori is simply knot insertion that does not change the surface. So, the simple scheme outlined above is preferable.

\section{Generalized bi-cubic subdivision}

Since we only locally improve Catmull-Clark subdivision, all input meshes suitable for Catmull-Clark subdivision will be admissible. In particular, global boundaries and $n$-sided facets are covered by Catmull-Clark rules. A designer wanting to treat a vertex with polar subdivision, has to configure its neighborhood as a polar structure (Section 1.1). A typical scenario is a cylindrical extrusion surrounded by 5 -sided facets. Given an input mesh and the maximal subdivision level $k$, generalized bi-cubic subdivision for Catmull-Clark meshes augmented with polar 

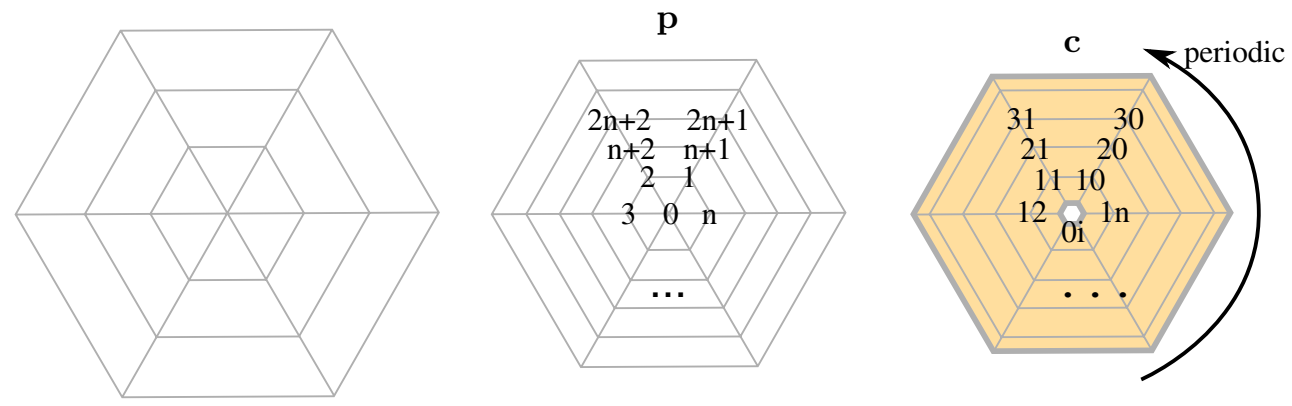

Figure 8. Finite polar capping by a periodic singular NURBS patch. (left) Initial polar structure, (middle) polar mesh with vertices $\mathrm{p}_{i}$ after one subdivision; (right) bi-cubic NURBS representation with control points $\mathrm{c}_{i j}$ : the collapsed center $\mathrm{c}_{0 i}$ and the exterior boundary are associated with 4-fold knots.

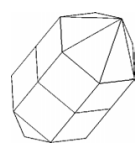

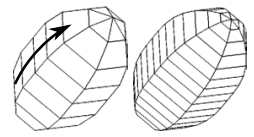

radial

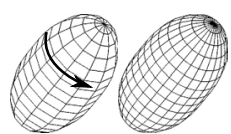

circular

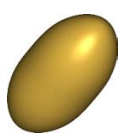

Figure 7. Mesh refinement preserving the limit surface of bi-cubic polar subdivision. From left to right: original mesh, two applications of radial subdivision, followed by two applications of circular subdivision, and limit surface.

structures is straightforward (see Figure 5).

1. Split off polar structures: Copy all the polar structures and remove polar vertices from the input mesh.

2. Subdivide polar structures: For each polar structure,

(a) subdivide $k$ times radially, and then

(b) subdivide $k$ times in the circular direction.

3. Subdivide the remaining mesh: Apply $k$ steps of Catmull-Clark subdivision to the mesh without the polar vertices.

4. Merge results: Drop the boundary facets of the meshes subdivided in steps 2 and 3 and join them by identifying the resulting boundary vertices.

By splitting the mesh into overlapping pieces, we introduced new boundaries in addition to any global boundaries of the input mesh. Subdivision steps 2 and 3 deal with these boundaries by dropping the vertices that do not have enough neighbors to apply the regular rules.

If a designer placed polar vertices too close together, or did not separate extraordinary limit points of CatmullClark subdivision, or created polar points of low valence,

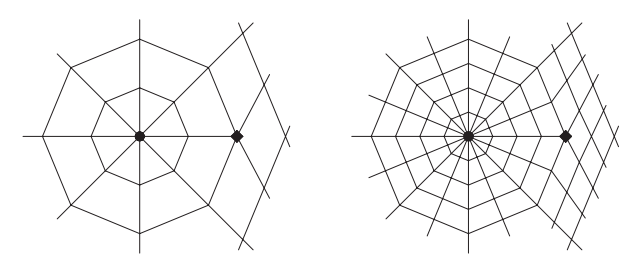

Figure 9. Separating Catmull-Clark and polar extraordinary limit points.

then applying step 3 and standard bi-cubic subdivision also in the 0-layer of the polar vertex (by interpreting triangular facets as degenerate quadrilaterals) doubles the valence and separates extraordinary limit points (see Figure 9). For this one initial step, computing the polar vertex with weight $\alpha:=\frac{3}{8(1-\beta)}-\frac{1}{2}$ yields good shapes for a range of $\beta$. We suggest $\beta:=\frac{1}{2}$ and $\alpha=\frac{1}{4}$.

\section{Finite bi-cubic constructions}

A related, different but complementary generalization of the bi-cubic setting is to model areas deviating from the tensor-product setting by a few bi-cubic NURBS patches. Since PCCM [12] gives a construction for Catmull-Clark layouts, we focus here on constructing a single bi-cubic spline for a polar structure. Just as PCCM yields a finite bi-cubic surface that is at least $C^{1}$ everywhere, the single bi-cubic NURBS surface will be $C^{1}$. Despite its singular parametrization at the central polar point, it can be shown to have bounded curvature. On input of a polar structure, the finite bi-cubic NURBS construction has the following steps (cf. Figure 8).

(i) (recommended for better shape) Subdivide the polar structure. Subdivide radially, twice for elongated ex- 
amples like tips of fingers. The resulting mesh $\mathbf{p}$ is labeled as in Figure 8, middle.

(ii) Convert the polar structure to a spline mesh. Initialize $\mathbf{c}_{i j}:=\mathbf{p}_{(i-1) n+j+1}$ for $i>1$ and $j=0, \ldots, n-1$. Both $u$ and $v$ knot sequences are uniform. The circular direction with parameter $u$ is periodic.

(iii) Interpolate the extraordinary limit point of bi-cubic polar subdivision. For $i=0, \ldots, n-1$, set

$$
\mathbf{c}_{0 i}:=\eta \mathbf{p}_{0}+(1-\eta) \frac{1}{n} \sum_{j=1}^{n} \mathbf{p}_{j}, \eta:=\frac{4(1-\beta)}{3},
$$

the limit formula derived in [7], and change the start of the radial knot sequence to a 4-fold knot associated with $\mathbf{c}_{0 i}$.

(iv) Match the limit normal of bi-cubic polar subdivision We project the neighbors of the central point into a common plane. For $i=0, \ldots, n-1$,

$$
\begin{gathered}
\mathbf{c}_{1 i}:=\mathbf{c}_{0 i}+2 \sigma \sum_{j=0}^{n-1} \Gamma_{j-i} \mathbf{p}_{j+1}, \quad \sigma_{\text {default }}:=\frac{3}{4}, \\
\Gamma_{k}:=\frac{1}{n} \cos \left(\frac{2 \pi k}{n}\right) .
\end{gathered}
$$

The projection of the spline coefficients does not alter the inherent $C^{2}$ continuity apart from the singularity at the extraordinary limit point; and the projection maps all radial tangents into the same plane with normal direction $\left(\mathbf{c}_{11}-\mathbf{c}_{00}\right) \times\left(\mathbf{c}_{12}-\mathbf{c}_{00}\right)$ at the extraordinary limit point.

(v) (optional) Additional knot insertion. It is common to have cubic NURBS patches with four-fold end knots. Knot insertion at the outer boundary yields e.g. $0,0,0,0,1,2, \ldots, m-1, m, m, m, m$ for the radial knots. The circular knot sequence remains uniform due to periodicity.

Figure 10 and 11, right, show examples of the NURBS construction. The spline surface is $C^{0}$ due to the common interpolated control vertex $\mathbf{c}_{00}$ that represents a collapsed edge $\mathbf{c}_{0 i}:=\mathbf{c}_{00}, i=0, \ldots, n-1$. The surface is singularly parametrized. Since singularly parametrized surfaces are commonly used in CAD applications, such packages handle and display the NURBS patch without problems. However, singularly parametrized surfaces are tricky to analyze $[10,11,14,2,16]$. The classical approach is an algebraic reparametrization of the surface in the singular point. In the Appendix, we use a novel approach that only becomes natural due to improved understanding of subdivision surfaces: we re-parametrize by a subdivision scheme that traces out the same surface as the NURBS patch. We find that the surface is $C^{1}$ and curvature bounded.

\section{Summary and Discussion}

Bi-cubic polar subdivision augments the capabilities of existing Catmull-Clark implementations. The extension is particularly valuable for extruded features and naturally complements Catmull-Clark in regions of high-valence. We propose compatible polar mesh refinement to minimally modify the existing infrastructure and add the good shape and simplicity of bi-cubic polar subdivision.

We also developed a finite polar spline generalization of standard bi-cubic splines. Pleasantly, this construction consists of a single NURBS patch. The representation is easy to add to existing CAD and animation modeling packages and is suitable for evaluation on the GPU. The central singularity presents no problem for rendering since the explicit normal is known and the Appendix shows that the surface curvatures are bounded.

The analysis of the finite construction in the Appendix defines and uses another polar subdivision scheme, called pbs. This raises the question whether we could use pbs in place of bi-cubic polar subdivision and thereby obtain a unified finite-plus-subdivision representation. We consider pbs less practical since it has a large subdivision footprint, with special rules for every $i$-link for $i=0,1,2,3$. Moreover, the generating functions associated with the 1 -link vertices are dependent and a special first step is required without which the convex hull property is not guaranteed.

All four surface types of Table 1 are compatible with one another in that their transitions are identical bi-cubic splines. The resulting surfaces are piecewise bi-cubic, $C^{2}$ almost everywhere and $C^{1}$ at isolated points (curves in the case of PCCM). Both the subdivision and the NURBS construction give equally valid meaning to the input mesh created and manipulated by the designer. And, by increasing the subdivision level, the resulting surfaces can be made arbitrarily close to allow switching from one modeling paradigm to the other. Conveniently, the polar pieces of each approach can be implemented as a simple extension of existing modeling tools.

\section{References}

[1] U. H. Augsdörfer, N. A. Dodgson, and M. A. Sabin. Tuning subdivision by minimising gaussian curvature variation near extraordinary vertices. Computer Graphics Forum (Proc. Eurographics), 25(3):263272, 2006.

[2] H. Bohl and U. Reif. Degenerate Beźier patches with continuous curvature. Computer Aided Geometric Design, 14(8):749-761, 1997. 
[3] E. Catmull and J. Clark. Recursively generated Bspline surfaces on arbitrary topological meshes. Computer Aided Design, 10:350-355, 1978.

[4] I. Ginkel and G. Umlauf. Loop subdivision with curvature control. In A. Scheffer and K. Polthier, editors, Proceedings of Symposium on Geometry Processing, June 26-28 2006, Cagliari, Italy, pages 163172. ACM Press, 2006.

[5] M. Halstead, M. Kass, and T. DeRose. Efficient, fair interpolation using Catmull-Clark surfaces. Proceedings of SIGGRAPH 93, pages 35-44, Aug 1993.

[6] K. Karčiauskas, A. Myles, and J. Peters. A $C^{2}$ polar jet subdivision. In A. Scheffer and K. Polthier, editors, Proceedings of Symposium on Geometry Processing, June 26-28 2006, Cagliari, Italy, pages 173180. ACM Press, 2006.

[7] K. Karčiauskas and J. Peters. Bicubic polar subdivision. ACM TOG, pages $\mathrm{xx}-\mathrm{xx}, 2007$. to appear.

[8] K. Karčiauskas, J. Peters, and U. Reif. Shape characterization of subdivision surfaces - case studies. Computer Aided Geometric Design, 21(6):601-614, july 2004.

[9] A. Levin. Modified subdivision surfaces with continuous curvature. In SIGGRAPH '06: ACM SIGGRAPH 2006 Papers, pages 1035-1040, New York, NY, USA, 2006. ACM Press.

[10] M. Neamtu and P. R. Pfluger. Degenerate polynomial patches of degree 4 and 5 used for geometrically smooth interpolation in $R^{3}$. Computer Aided Geometric Design, 11(4):451-474, 1994.

[11] J. Peters. Parametrizing singularly to enclose vertices by a smooth parametric surface. In in: S. MacKay and E. M. Kidd, editors, Graphics Interface '91, pages 17, Calgary, Alberta, 1991. Canadian Information Processing Society.

[12] J. Peters. Patching Catmull-Clark meshes. In SIGGRAPH 'O0: Proceedings of the 27th annual conference on Computer graphics and interactive techniques, pages 255-258, New York, NY, USA, 2000. ACM Press/Addison-Wesley Publishing Co.

[13] J. Peters and U. Reif. Shape characterization of subdivision surfaces: basic principles. Computer Aided Geometric Design, 21(6):585-599, 2004.

[14] U. Reif. A note on degenerate triangular Beźier patches. Comput. Aided Geom. Des., 12(5):547-550, 1995.
[15] U. Reif. A unified approach to subdivision algorithms near extraordinary vertices. Computer Aided Geometric Design, 12:153-174, 1995.

[16] U. Reif. TURBS-topologically unrestricted rational $B$-splines. Constructive Approximation. An International Journal for Approximations and Expansions, 14(1):57-77, 1998.

[17] U. Reif and J. Peters. Structural analysis of subdivision surfaces - a summary. In K. Jetter et al., editor, Topics in Multivariate Approximation and Interpolation, volume 12, pages 149-190, 2005.

[18] S. Schaefer and J. D. Warren. On $\mathrm{C}^{2}$ triangle/quad subdivision. ACM Transactions on Graphics, 24(1):28-36, 2005.

[19] J. Stam and C. T. Loop. Quad/triangle subdivision. Computer Graphics Forum, 22(1):79-86, 2003.

\section{Appendix}

To analyze the singularly parametrized polar NURBS patch with coefficients $\mathbf{c}_{i j}$ defined in Section 4, we derive a new projected bi-cubic subdivision (pbs). Pbs traces out the same surface as the NURBS patch so that we can analyze the NURBS patch by analyzing pbs. We start by showing that bi-cubic spline subdivision of the NURBS patch does not yield a useful analysis.

\subsection{Failure of standard subdivision of the polar spline.}

We focus on the $i$-links of the polar vertex $\mathbf{c}_{00}$ for $i=0,1,2,3$, and initialize for subdivision level $m=0$, $\mathbf{c}_{k i}^{m}:=\mathbf{c}_{i j}$ for $j=0, \ldots, n-1, k=0,1,2,3$. If the radial curve with coefficients $\left[\begin{array}{llll}\mathbf{c}_{0 i}^{m} & \mathbf{c}_{1 i}^{m} & \mathbf{c}_{2 i}^{m} & \mathbf{c}_{3 i}^{m}\end{array}\right]^{\mathrm{t}}$ has initially the knot sequence $0,0,0,0,1,2,3,4$, the standard cubic subdivision amounts to inserting knots at $1 / 2$ and $3 / 2$, i.e. to applying the curve subdivision matrix

$$
\left[\begin{array}{l}
\mathbf{c}_{0 i}^{m+1} \\
\mathbf{c}_{1 i}^{m+1} \\
\mathbf{c}_{2 i}^{m+1} \\
\mathbf{c}_{3 i}^{m+1}
\end{array}\right]=\left[\begin{array}{cccc}
1 & 0 & 0 & 0 \\
1 / 2 & 1 / 2 & 0 & 0 \\
0 & 3 / 4 & 1 / 4 & 0 \\
0 & 3 / 16 & 11 / 16 & 1 / 8
\end{array}\right]\left[\begin{array}{c}
\mathbf{c}_{0 i}^{m} \\
\mathbf{c}_{1 i}^{m} \\
\mathbf{c}_{2 i}^{m} \\
\mathbf{c}_{3 i}^{m}
\end{array}\right] .
$$

The radial subdivision matrix of the NURBS patch combines $n$ curve subdivision matrices. Since an arbitrary B-spline with one end collapsed to an interpolated vertex needs not be $C^{1}$, the spectral analysis of this subdivision must certainly fail. The apparently $n$-fold eigenvalue of 1 becomes a single leading eigenvalue by the standard approach of distributing the contribution $\mathbf{c}_{0 i}=\mathbf{c}_{00}$ evenly with weight $1 / n$. However, there are also $n$ subdominant eigenvalues of size $1 / 2$ that, off hand, contradict the uniqueness of a tangent plane. In general, such a scheme is not $C^{1}$. 


\subsection{Projected subdivision of the polar spline.}

Construction step (iv) in Section 4 maps the 1-link into a unique plane. To use this projection in the analysis, we say the 1-link vertices $\mathbf{c}_{1 i}$ are in oval position if there exist two linearly independent vectors $\mathbf{e}_{1}$ and $\mathbf{e}_{2}$ such that

$$
\mathbf{c}_{1 i}=\mathbf{c}_{00}+\mathbf{e}_{1} \cos (2 \pi i / n)+\mathbf{e}_{2} \sin (2 \pi i / n) .
$$

That is, the 1-link is equally distributed on an oval, the affine projection of a circle centered at the extraordinary limit point $\mathbf{c}_{00}$, and lies in the plane containing $\mathbf{c}_{00}$ and spanned by $\mathbf{e}_{1}$ and $\mathbf{e}_{2}$. We observe that,

- if the control points $\mathbf{c}_{1 i}^{m}$ are in oval position, row two of Equation 4 assigns $\left(\mathbf{c}_{00}^{m}+\mathbf{c}_{1 i}^{m}\right) / 2$ to $\mathbf{c}_{1 i}^{m+1}$ so that the points $\mathbf{c}_{1 i}^{m+1}$ are also in oval position.

- Equation 3, in the form

$$
\mathbf{c}_{1 i}^{m} \leftarrow \mathbf{c}_{00}^{m}+2 \sigma \sum_{j=0}^{n-1} \Gamma_{j-i} \mathbf{c}_{1 j}^{m}
$$

maps a 1-link $\mathbf{c}_{1 i}$ into oval position. Applying this projection (6) with $\sigma=1$ to Equation 5 shows that a 1-link in oval position remains unchanged under the projection.

We now define the projected bi-cubic subdivision (pbs) scheme to be standard bi-cubic subdivision with the second row in Equation 4 modified by applying the projection (6):

$$
\begin{aligned}
\mathbf{c}_{1 i}^{m+1} & =\frac{1}{2} \mathbf{c}_{0 i}^{m}+\frac{1}{2}\left(\mathbf{c}_{0 i}^{m}+2 \sum_{j=0}^{n-1} \Gamma_{j-i} \mathbf{c}_{1 j}^{m}\right) \\
& =\mathbf{c}_{0 i}^{m}+\sum_{j=0}^{n-1} \Gamma_{j-i} \mathbf{c}_{1 j}^{m} .
\end{aligned}
$$

Lemma 1. The singular NURBS patch defined in Section 4 is identical to the limit surface generated when pbs is applied to its control points.

Proof. By construction of the NURBS patch, the 1-link $\mathbf{c}_{1 j}^{0}$ is in oval position. For a 1-link $\mathbf{c}_{1 j}$ in oval position, Equation 7 and row two of Equation 4 yield identical new 1links.

\subsection{Analysis of projected bi-cubic subdivi- sion}

A standard analysis below shows pbs to generate $C^{1}$ surfaces with bounded curvature. The usual caveat that control points should be generic ([17], pg. 164) applies to the mesh with the union of $\mathbf{c}_{0 i}$ interpreted as a single point.

Lemma 2. Surfaces generated by the pbs scheme are $C^{1}$ and have bounded curvature.
Proof. We split the contribution of $\mathbf{c}_{00}$ evenly amongst the $\mathbf{c}_{0 i} i \in 0, \ldots, n-1$ and write the pbs circulant matrix as

$$
\left[\begin{array}{c}
\vdots \\
\mathbf{c}_{0 i}^{m+1} \\
\mathbf{c}_{1 i}^{m+1} \\
\mathbf{c}_{2 i}^{m+1} \\
\mathbf{c}_{3 i}^{m+1} \\
\vdots
\end{array}\right]=\left[\begin{array}{cccc}
A_{0} & A_{1} & \ldots & A_{n-1} \\
A_{n-1} & A_{0} & \ldots & A_{n-2} \\
\vdots & & \ddots & \vdots \\
\dot{A_{1}} & \ldots & A_{n-1} & \dot{A}_{0}
\end{array}\right]\left[\begin{array}{c}
\vdots \\
\mathbf{c}_{0 i}^{m} \\
\mathbf{c}_{1 i}^{m} \\
\mathbf{c}_{2 i}^{m} \\
\mathbf{c}_{3 i}^{m} \\
\vdots
\end{array}\right]
$$

where for $i>0$,

$$
A_{0}:=\left[\begin{array}{cccc}
1 / n & 0 & 0 & 0 \\
1 / n & \Gamma_{0} & 0 & 0 \\
0 & 3 / 4 & 1 / 4 & 0 \\
0 & 3 / 16 & 11 / 16 & 1 / 8
\end{array}\right], A_{i}:=\left[\begin{array}{cccc}
1 / n & 0 & 0 & 0 \\
1 / n & \Gamma_{i} & 0 & 0 \\
0 & 0 & 0 & 0 \\
0 & 0 & 0 & 0
\end{array}\right] .
$$

Under discrete Fourier transform, $\hat{A}_{i}:=\sum_{j=0}^{n-1} w^{i j} A_{j}$, $w^{i j}:=\exp (2 \pi i j \sqrt{-1} / n)$, the circulant system diagonalizes into $\operatorname{diag}\left(\hat{A}_{0}, \ldots, \hat{A}_{n-1}\right)$ where $\hat{\Gamma}_{i}:=\sum_{j=0}^{n-1} w^{i j} \Gamma_{j}$ and

$$
\hat{A}_{0}:=\left[\begin{array}{cccc}
1 & 0 & 0 & 0 \\
1 & \hat{\Gamma}_{0} & 0 & 0 \\
0 & 3 / 4 & 1 / 4 & 0 \\
0 & 3 / 16 & 11 / 16 & 1 / 8
\end{array}\right], \hat{A}_{i}:=\left[\begin{array}{cccc}
0 & 0 & 0 & 0 \\
0 & \hat{\Gamma}_{i} & 0 & 0 \\
0 & 3 / 4 & 1 / 4 & 0 \\
0 & 3 / 16 & 11 / 16 & 1 / 8
\end{array}\right] \text {. }
$$

The eigenvalues of $\hat{A}_{0}$ are $1, \hat{\Gamma}_{0}, 1 / 4,1 / 8$, and those of $\hat{A}_{i}$ are $0, \hat{\Gamma}_{i}, 1 / 4,1 / 8$. Since $\hat{\Gamma}_{0}=0$ and $\hat{\Gamma}_{1}=\hat{\Gamma}_{n-1}=1 / 2$, while $\hat{\Gamma}_{i}=0$ in all other cases, the subdominant eigenvalue is $\lambda:=1 / 2$ and corresponds to Fourier blocks $\hat{A}_{1}$ and $\hat{A}_{n-1}$. The left eigenvector of $\hat{A}_{1}$ corresponding to eigenvalue $1 / 2$ is $(0,1,3,6)$. Therefore the characteristic map $\mathbf{r}$ (labeled using the same indexing as $\mathbf{c}$ in Figure 8, right) has coefficients

$$
\left(\mathbf{r}_{0 i}, \mathbf{r}_{1 i}, \mathbf{r}_{2 i}, \mathbf{r}_{3 i}\right):=\left(\mathbf{0}, \mathbf{s}_{i}, 3 \mathbf{s}_{i}, 6 \mathbf{s}_{i}\right), \quad \mathbf{s}_{i}:=\left[\begin{array}{c}
\cos \left(\frac{2 \pi i}{n}\right) \\
\sin \left(\frac{2 \pi i}{n}\right)
\end{array}\right] .
$$

The characteristic map is regular for arbitrary valences and satisfies the injectivity criterion of Theorem 3.5 of [15], so that the limit surface is $C^{1}$ as claimed. The subsubdominant eigenvalue $\mu:=1 / 4=\lambda^{2}$ comes from independent Fourier blocks, indicating that it has equal algebraic and geometric multiplicity. Hence by Theorem 3.3 of [13], the surface has bounded curvature.

By Lemma 1, standard bi-cubic subdivision and projected bi-cubic subdivision generate the same surface, and Lemma 2 implies the hoped-for theorem.

Theorem 3. A NURBS patch constructed according to Section 4 is $C^{1}$ and has bounded curvature at the central point. 

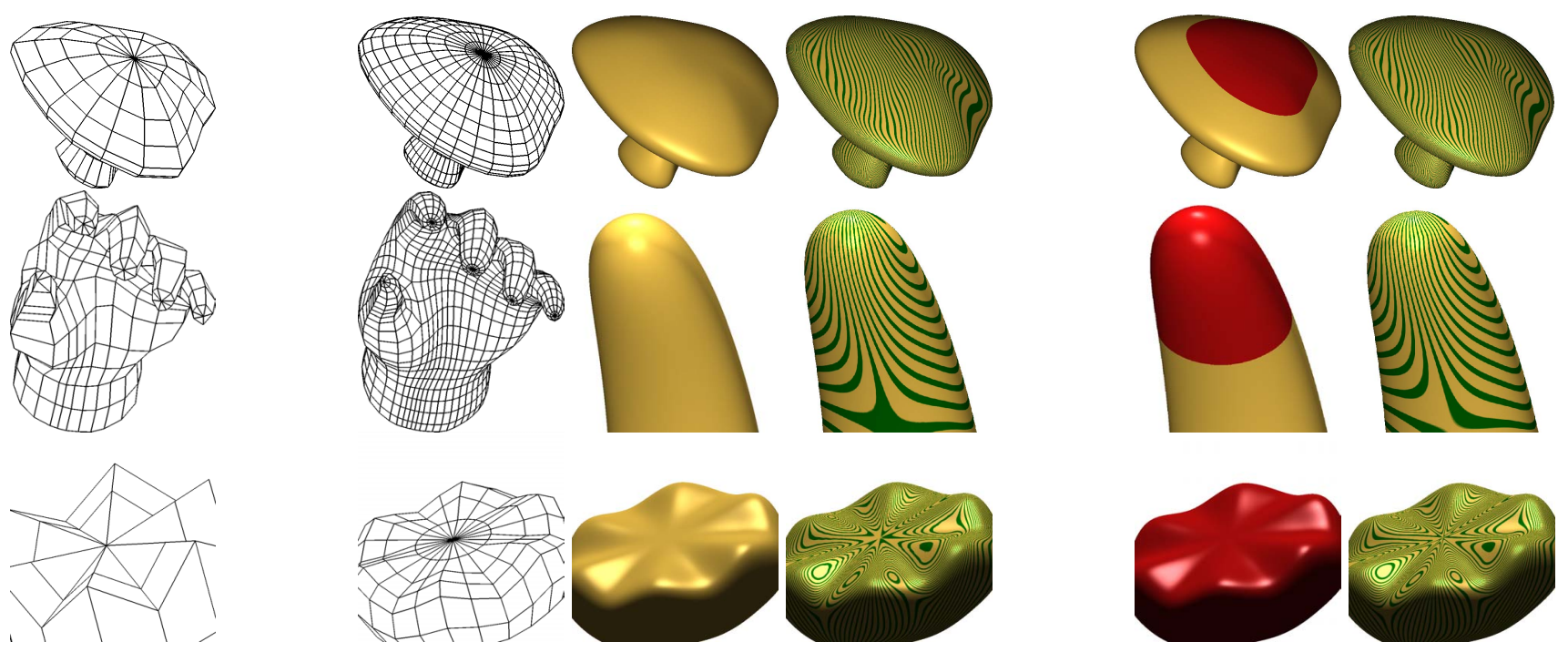

Figure 10. Results. From left to right: control mesh, mesh after one subdivision, subdivision limit surface, highlight lines on subdivision surface, finite NURBS construction, finite NURBS construction highlight lines. In row two, we zoom in on one of the fingers. Row three illustrates a high-valent saddle.
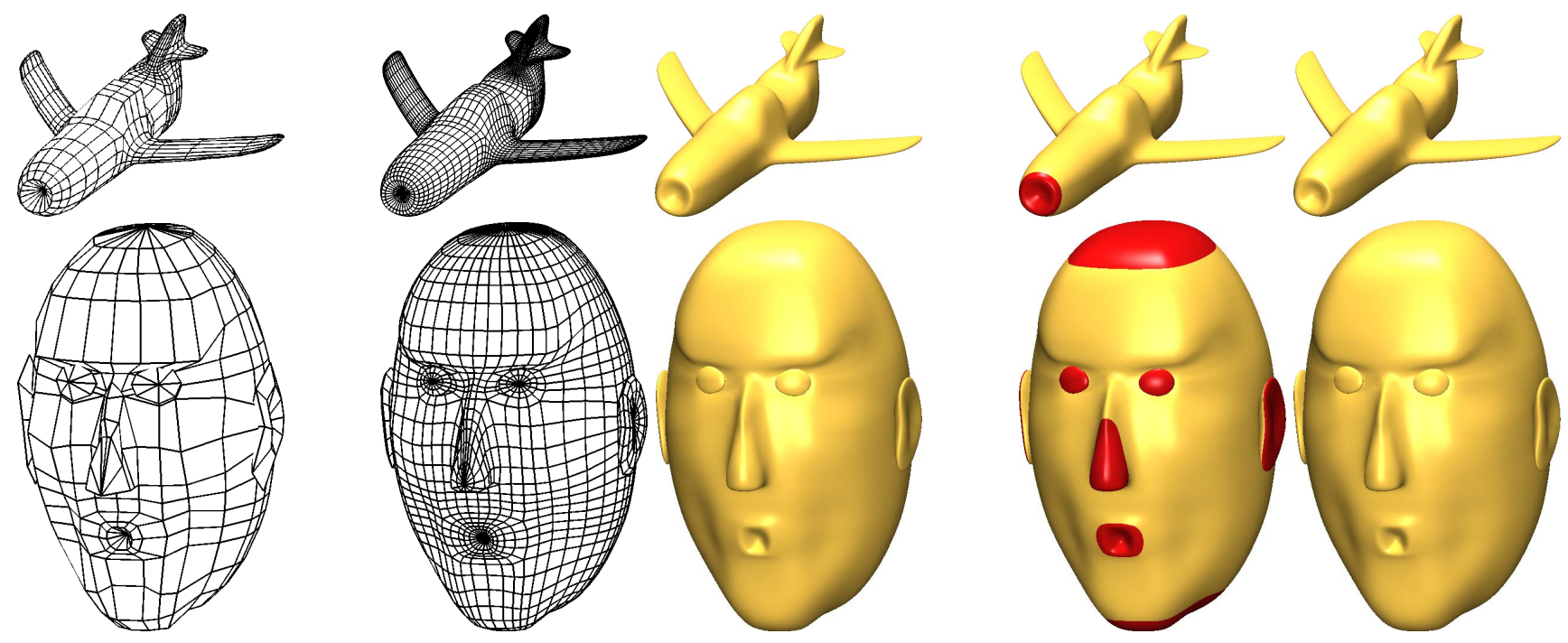

Figure 11. Results. From left to right: control mesh, mesh after one subdivision, subdivision limit surface, polar regions, finite NURBS construction. 